\title{
Structural and electronic properties of the P3HT-PCBM dimer: A theoretical Study
}

Israel Gutiérrez-González, ${ }^{1,4}$ Bertha Molina-Brito, ${ }^{2}$ Andreas W. Götz, ${ }^{3}$ F. L. CastilloAlvarado, ${ }^{1} \quad$ and Juan I. Rodríguez, ${ }^{1 *}$

${ }^{1}$ Escuela Superior de Fîsica y Matemáticas, Instituto Politécnico Nacional, Edificio 9, U.P. A.L.M.,

Col. San Pedro Zacatenco, C.P. 07738, México D.F., México.

${ }^{2}$ Facultad de Ciencias, Universidad Nacional Autónoma de México, Apartado Postal 70-646, 04510 México D.F., México.

${ }^{3}$ San Diego Supercomputer Center, University of California San Diego, 9500 Gilman Drive, La Jolla, CA, 92093-0505, USA.

${ }^{4}$ Unidad Académica Profesional Nezahualcóyotl, Universidad Autónoma del Estado de México, Av. Bordo de Xochiaca s/n C.P. 57000, Ciudad Nezahualcóyotl, Edo. de México, México.

\section{ABSTRACT}

A density functional theory (DFT) study of the supramolecular dimer formed by a 8-unit oligomer of the poly(3-hexylthiophene) (P3HT) and the fullerene derivative $[6,6]$-phenyl$\mathrm{C}_{61}$-butyric acid methyl ester (PCBM) is presented. A dispersion-corrected exchangecorrelation potential was used to afford for the long-range van der Waals interactions. Our calculations predict two stable isomers in which the P3HT 8-mer forms a U-shaped structure surrounding the PCBM. From time-dependent DFT calculations it was determined that the maximum absorption intensity of the P3HT-PCBM dimer undergoes a blue-shift of about $80 \mathrm{~nm}$ with respect to isolated the P3HT oligomer in qualitative agreement with the experimental facts.

* Corresponding author: Juan I. Rodriguez. E-mail: juan@esfm.ipn.mx 


\section{Introduction}

In addition of being a renewable and non-polluting energy source, organic or plastic solar cells have attracted much attention as being an unexpensive, flexible and lightweight device. [1-2] The organic "bulk heterojunction" (BHJ) solar cells made of blends of an electron donor (usually a $\pi$-conjugate polymer like P3HT) and an electron acceptor (usually a fullerene derivative like $\mathrm{PCBM})$ are among the most efficient $(\eta \simeq 5 \%)$ organic cells reported to date. [3-8] It has been determined that the BHJ solar cell efficiency depends on important processes like exciton formation and the charge transfer mainly at the interface P3HT/PCBM. $[1-3,9-28]$ Yet there is not a complete understanding of how the P3HT and PCBM are interacting at the molecular level and on what kind of structure they form.

In order to cast some light on how the PCBM and P3HT interact at the molecular level we have performed Kohn-Sham density functional theory (DFT) calculations on the supramolecular dimer formed by a 8 -unit oligomer (8-mer) of P3HT and the PCBM in the gas phase. A partial search of the dimer potential energy surface was performed to determine stable structures. Time dependent DFT (TDDFT) calculations have been performed to obtain the absorption spectra of the P3HT-PCBM dimer and the isolated systems. The remainder of the article is organized as follows: The computational methodology is introduced in Sec. II., which describes the details of computational 
calculations performed in our study. Our results and conclusions are shared in Secs. III and IV , respectively.

\section{Computational Methodology}

In order to "partially" explore the potential energy surface (PES) of the supramolecular P3HT-PCBM dimer in the gas phase, and using information from previous calculations on similar supramolecular systems, [29-31] a family of at least 10 different nuclear geometries of the dimer P3HT-PCBM were constructed to be used as initial structures in the geometry optimizations. The optimized structures of the isolated molecules (P3HT-8mer and PCBM) were used to construct the starting geometries for the dimer optimizations (see Figure 1 (b)

) changing the relative position of $\mathrm{P} 3 \mathrm{HT}$ and $\mathrm{PCBM}$ but initially restraining the "intermolecular" distance to about 3-5A (the closest distance between rings of each system). As a result of this process, two different stable isomers of the P3HT-PCBM dimer were obtained. Their structural and electronic properties are described in detail in the next section. A P3HT oligomer with 8 units was chosen as the largest oligomer to model the P3HT polymer (which is used in the experiment) that make a P3HT-PCBM dimer calculation feasible within the DFT framework. Other oligomer sizes have been considered. [11,31] All DFT and TDDFT calculations were performed with the Amsterdam Density Functional (ADF 
2013) package. [35-37] The geometries were fully optimized at the DFT level considering a convergence threshold for the energy gradients with respect to the nuclear coordinates equal to $10^{-4}$ Hartree/Angstrom. In order to properly take into account the long-range van der Waals interactions, the Grimme's dispersion-corrected version [32-33] of the (generalized-gradient-approximation) Perdew-Burke-Ernzerhof exchange-correlation (xc) potential [34] was used (PBE-D3). A triple- $\zeta$ polarized Slater (TZP-all electron) basis set from the ADF basis library was used in all calculations. The ADF integration parameter was set to 6 in order to ensure small errors in solving the KS equations and computing the dimer properties. Unless otherwise stated, the ADF default settings for the SCF procedure, geometry optimizations and property calculations were used. [35-37] TDDFT calculations using the same xc potential (and all other parameters) were performed to obtain absorption spectra of both the isolated molecules and the P3HT-PCBM dimer at the DFT optimized geometries. The lowest 250 and 200 singlet-singlet allowed transitions were computed for the isolated PCBM and P3HT, respectively. For the dimer isomers, however, only 150 allowed transitions were computed due to memory demand. To simulate the absorption spectra, a superposition of $100 \mathrm{~nm}$-width Gaussian functions was considered.

\section{Results and Discussion}

Figure 1 shows two stable isomer structures (with their energies) of the P3HT-PCBM dimer, 
along with the two initial geometries used in the geometry optimization. All other 8 initial geometries used in geometry optimizations either converged to the one of the isomers shown in Figure 1 or diverged (the P3HT starts going away from PCBM). As can be seen from Figure 1, in both isomers the P3HT tends to "embrace" the PCBM. In order to better understand these isomer structures, Figure 1 (c) shows the same structures but displaying only the P3HT 8-mer backbone and hiding all hydrogen atoms which allows one to see more clearly that the P3HT 8-mer adopts an U-shape (or horseshoe shape) structure surrounding the PCBM (in both isomers). P3HT embraces the PCBM more uniformly and more closely in the region of the center of P3HT for both isomers. ${ }^{1}$ It is worth mentioning that the alkane side chains on the P3HT get also bent toward the PCBM in both isomers. In order to better determine the role of the Grimme van der Waals correction, we performed a geometry optimization using the same initial geometry but using the PBE functional without the Grimme correction. The geometry did not converge, the P3HT did not get attracted by PCBM to lower the energy. It indicates that the van der Waals intermolecular interaction, taken into account via Grimme correction of the xc functional, is playing a crucial role for the dimer to adopt the stable structure shown in Figure 1 (see discussion below). No covalent bonds are formed between P3HT and PCBM. The minimun distance between an

1 These type of $U$-shape structures are usually found in supramolecular systems that consider intermolecular interactions, like protein-protein interactions, etc.. 
atom on the P3HT and one on the PCBM is equal to 2.08A and 2.27A for Isomer 1 (I1) and Isomer 2 (I2), respectively (see Figure 2). From Figure 2 notice that the populations of interatomic distances between P3HT and PCBM atoms in the range of 2 to $3 \mathrm{~A}$ are greater in I1 than in I2, which indicates that there are regions in space where P3HT and PCBM get closer in I1 than in I2. It can be observed rotations $\left(\sim 15^{\circ}\right)$ of the P3HT thiophene rings around an axes "parallel" to the backbone. These rotations happen in a alternate way: one ring is rotated clockwise and the adjacent ring counter clockwise and so on. The dihedral angles between adjacent thiophene rings in the P3HT chain in-the-dimer can be seen in Figure 3. The high value of $\mathrm{D} 6=334^{\circ}$ in Isomer 2 indicates the high torsion $\left({ }^{\sim} 154^{\circ}\right)$ that undergoes the thiofene ring R7 in P3HT which does not happen in Isomer 1. We believe that this high torsion is because the relative position of the butyric acid group in the Isomer 2 with respect to rings R6 and R7. From Figure 1 we can see that this group seems to "cross" the (virtual) surface defined by the P3HT backbone (see Figure 1(b)-(c)) which makes the carboxylic group to get close (3.6-4A) to rings R6 and R7. The high rotation of R7 makes it to get in a head-head position with R6 breaking the global head-tail arrangement between two adjacent rings in the P3HT chain breaking so the local translational symmetry (see Figures 1 and 3). In Isomer 1, however, the situation is quite different. The butyric acid group in Isomer 1 seems to line up with the surface defined by the backbone of P3HT (getting parallel to this surface). The ring R7 in this case undergoes a rotation similar to the 
other rings. The head-tail arrangement of the thiophene rings in Isomer 1 holds. Repercussion of this destruction of the ordering in P3HT on the optical properties of the P3HT-PCBM dimer isomers is discussed below. In this work we are mostly interested in determining stable structures of the P3HT-PCBM dimer and in casting some light onto the relation structure/optical-properties within the DFT framework.

The computed value of the HOMO-LUMO gap for the gas-phase isolated PCBM, $\mathrm{Eg}=1.48 \mathrm{eV}$, is in relative agreement with the theoretical value reported by Thompson et al. $(1.4 \mathrm{eV})[38]$ and the experimental value reported by Cook et al. $(1.8 \mathrm{eV})[16]$. As for the gas-phase isolated P3HT -8 mer, our computed value for the energy gap, Eg $=1.31 \mathrm{eV}$, is also in relative agreement with the theoretical value reported by Drori et al. (1.49eV) [18] and the experimental value $\mathrm{E}_{\mathrm{exp}}=1.9 \mathrm{eV}[16]$. Thus our gas phase calculation at the DFT/PBE/TZP level of theory underestimates the experimental energy gap of the corresponding films by about $0.4-0.6 \mathrm{eV}$. The energy gap of the P3HT-PCBM dimer is equal to $\mathrm{Eg}=0.37 \mathrm{eV}$ and $\mathrm{Eg}=0.37 \mathrm{eV}$ for isomers 1 and 2, respectively. Notice that the gap for the dimer is reduced by about $70 \%$ for both isomers with respect to isolated P3HT computed both at the same level of theory. It is known that the GGA xc functionals tend to underestimate energy gaps [39-40] and that usually they are not that accurate to predict charge transfer excitation energies for some molecules. [41-42] Unfortunately it was not 
possible for us to perform TDDFT calculations using more suitable functionals for the TDDFT calculations, like the range--separated (RS) functionals, due mainly to the size of the system. ${ }^{2}$ Our election of the xc functional was made mainly to get the structural properties of the supramolecular dimer formed by the P3HT and PCBM. It has been shown that the PBE-D3 functional is suitable to study stable structures and interaction energies of supramolecular systems for which stacking interactions are important. [32-33] The criterion for the election of the xc functional for the TDDFT calculations was based on getting an equilibrium between quality and low computational cost. We believe, however, that the optical properties obtained at the TDDFT/PBE-D3/TZP level of theory at the PBE-D3 optimized geometries, which might be the only information in this respect for the P3HTPCBM dimer available to date, might be useful as long as one is aware of its predicting limitation (see below). ${ }^{3}$

Figure 4 shows the localization of the molecular orbitals (MO) for both isomers along with the principal allowed transitions predicted by our TDDFT calculations. Interestingly the occupied MO's, HOMO, HOMO-1 and HOMO-2 are localized mainly on the P3HT for both isomers. In the contrary, the LUMO, LUMO+1 and LUMO+2 are localized mainly on

2 Attempts to perform the TDDFT calculations using range-separated functionals as implemented in ADF were not successful due to high computational cost and very slow convergence in the Davidson algorithm.

3 TDDFT calculations considering fewer transitions and using the B3LYP xc functional with a DZP basis set reproduce the same qualitative features of the absorption spectra as obtained with the PBE functional. 
the PCBM also for both isomers, though some of the lowest allowed most intense transitions involve higher unoccupied molecular orbital as is discussed below. From Figure 5 we can see that the calculated spectrum for the PCBM reproduces qualitatively the main features reported from experimental results $[16,43]$, namely, it shows strong optical absorption predominantly in the UV region, with very weak absorption in the visible region (from 350nm-700nm, see the inset in Figure 5). Instead, the calculated spectrum for the P3HT oligothiophene shows a large red-shifted absorption profile compared with that of the pure P3HT films. At this point, it is worthwhile to mention that a red-shift has previously been reported for oligothiophene derivatives. [44-47] In our simulated spectrum appear two strongest absorption peaks at $685 \mathrm{~nm}$ and $780 \mathrm{~nm}$ which are mainly related to electronic transitions HOMO - LUMO+1 and HOMO - LUMO, respectively. A third absorption peak (b peak) is fashioned by the contributions of several electronic transitions: one weak $(f=$ $0.0505)$ located at $580 \mathrm{~nm}$, another more intense $(f=0.5374)$ at $553 \mathrm{~nm}$ and one very weak $(f$ $=0.0081)$ at $523 \mathrm{~nm}$, which represent a HOMO $-\mathrm{LUMO}+2, \mathrm{HOMO}-1-\mathrm{LUMO}+1$ and HOMO-1 - LUMO transitions, respectively. These three bands can be characterized as $\pi$ - $\pi *$ transitions on the backbone of the oligothiophene (see Figure 4a). In addition, at $476 \mathrm{~nm}$ and $470 \mathrm{~nm}$ we can see two weak transitions ( $f=0.0792$ and $f=0.0838$ respectively) yielded by HOMO-1 - LUMO+3 and HOMO-2 - LUMO+1 transitions. As reference, for pure P3HT films have experimentally been reported two maxima absorption peaks and one 
shoulder at $493 \mathrm{~nm}, 517 \mathrm{~nm}$ and $572 \mathrm{~nm}$ respectively, also attributed to the $\pi-\pi *$ transitions. [44-47] From the Figure 5 notice that our dimer (gas phase) spectra reproduce qualitatively the blue-shift of the maximum absorption intensity peaks reduction of the absorption in the red region obtained for blend P3HT:PCBM films and the depletion of the peaks compared with those of the P3HT. [44-46] This decreasing in intensity is stronger for I2 than for I1. Clearly the signal obtained for the oligothiophene at $780 \mathrm{~nm}$ is totally depleted for I2, however, for I1 the spectrum shows two weak absorption peaks at $839 \mathrm{~nm}$ and 748nm attributed to the transitions HOMO (localized on P3HT) - LUMO+5 (localized on PCBM) and HOMO - LUMO+7 (localized on P3HT), respectively (see Figure 4 (b)). Notice that the absorption peak at $685 \mathrm{~nm}$ in P3HT is retained for both isomers (at $696 \mathrm{~nm}$ for I1 and 683 for I2), but with diminution in the intensity of $40 \%$ and $74 \%$ for I1 and I2, respectively, compared with that of the P3HT. The spectrum for both dimer isomers retains the peak ( peak b) in the visible range, which is due to several electronic transitions. Interestingly the most intense transitions that contribute to peak $\mathrm{b}$ in the spectrum for I1 involve MO's localized on the P3HT (occupied MO's) and PCBM (unoccupied MO's), while for I2 the most intense transitions involve MO's predominantly localized on the P3HT (see Figure 4). Notice the blue-shift of about $80 \mathrm{~nm}$ of the maximun absorption intensity peaks and the overall depletion of the absorption intensity for the P3HT-PCBM dimer with respect to the spectrum of the isolated P3HT oligomer. Our TDDFT calculations on the dimer in 
the gas phase are capable of reproducing qualitatively well the experimentally reported blueshift of the maximum absorption intensity peaks (whom value depends on the relative composition P3HT:PCBM) and a decreasing of the absorption intensity for the P3HTPCBM blends with respect to pure P3HT films. [43-44] It is worth mentioning that this experimentally determined blue-shift of the the maximum absorption intensity peaks and the reduction of the red part of the absorption have been attributed to, first, the destruction of ordering in the P3HT chains in the presence of PCBM and, second, to a non-photoinduced charge transfer between P3HT and PCBM. [45,48] Our theoretical calculations on the P3HT-PCBM dimer in the gas phase might contribute to confirm this fact. According to our calculations, the 8-unit oligomer P3HT gets deformed in a U-shape structure destructing (up to certain degree) the ordering of the P3HT oligomer chain. If we compare the absorption spectrum of the dimer with the one of the isolated P3HT oligomer, it could be observed an overall blue-shift of the maximum absorption intensity peaks in the dimer with respect to the spectrum of the isolated P3HT oligomer. As previously discussed, the translational symmetry (that particular related to the ring head-tail arrangement) was partially broken in P3HT oligomer in Isomer 2 but not in Isomer 1 . Thus it could be considered that the ordering destruction of the P3HT chain in the Isomer 2 is more drastic. Coincidentally both effects the reduction of intensity in the red part and the blue-shift of the maximum absorption intensity peaks of the spectrum in the dimer Isomer 2 are also more 
drastic (see footnote 3). Using an ultrafast method developed by us [49] aimed to be applied to large molecules, [50] we computed the Bader's charges for P3HT and PCBM in each isomer: $q_{P 3 H T}^{I I}=0.097, q_{P C B M}^{I I}=-0.098$; and $\quad q_{P 3 H T}^{I 2}=0.010 ; q_{P C B M}^{I 2}=-0.008{ }^{4}$ whom values indicates a charge transfer between P3HT and PCBM particularly in Isomer 1.

\section{Conclusions}

We have performed Kohn-Sham DFT calculations for the supramolecular dimer formed by the 8-unit oligomer of the P3HT and the PCBM. The calculations were performed in the gas phase using the the Grimme's dispersion-corrected version [32-33] of the (generalizedgradient-approximation) Perdew-Burke-Ernzerhof exchange-correlation（xc）potential [34]. Considering up to 10 initial geometries of the P3HT-PCBM dimer in a geometry optimization procedure, it was possible to obtain two stable isomers of the P3HT-PCBM dimer (as far as we know by the first time). In both dimer isomers the P3HT tends to "embraces" the PCBM adopting a U-shape structure stabilizing the system. The van der Waals interaction is playing a key role for the P3HT to adopt this U-shape form. The energy gap of the P3HT-PCBM of the dimer is reduced by about 70\% with respect to the isolated P3HT. TDDFT calculations show a reduction of intensity in the red part and a

4 The charges on P3HT and PCBM do not exactly sum to zero due to the numerical method employed. [49] 
blue-shift on the maximum absorption intensity peaks in the dimer spectrum with respect to the spectrum of the isolated P3HT oligomer. It could be determined a charge transfer between P3HT and PCBM according to Bader charges in both dimer isomers. These theoretical facts might contribute to confirm the experimentally determined blue-shift of the maximum absorption intensity peaks and the reduction of the red part in the absorption of P3HT-PCBM blends which have been attributed to the destruction of ordering in the P3HT chains in the presence of PCBM and to a non-photoinduced charge transfer between P3HT and PCBM. $[45,48]$

\section{Acknowledgments}

JIR thanks SIP-IPN (project 20144466) for financial support and IPN for the "Plaza de Excelencia”. JIR also gratefully acknowledge Prof. Paul Ayers' group at McMaster University for sharing the SHARCNET computer facilities. BMB is thankful for the computer facilities of the "Dirección General de Cómputo y Tecnologías de Información y Comunicación” (DGTIC, UNAM) and the support by PAPIIT-UNAM (IN119811). AWG acknowledges support by a TRO grant of the University of California San Diego and funding provided by the Department of Energy, Office of Biological and Environmental Research and Office of Advanced Scientific Computing Research (SciDAC award DE-AC36-99G0-10337). 
This work used the Extreme Science and Engineering Discovery Environment (XSEDE), which is supported by National Science Foundation grant number ACI-1053575. Computer time was provided by the San Diego Supercomputer Center through XSEDE awards TGCHE100149 and TG-CHE130010 to AWG. 


\section{REFERENCES}

1. S. S. Sun, N. S. Sariciftci (Editors), "Organic Photovoltaics. Mechanisms, Materials, and Devices” . Taylor \& Francis Group LLC, Florida (2005).

2. C. Brabec, V. Dyakonov, J. Parisi, N. S. Sariciftci (Editors), "Organics Photovoltaics". Springer-Verlag, Berlin (2003).

3. H. Hoppe and N. S. Sariciftci, "Bulk Heterojunction Solar Cells", in "Organic Photovoltaics. Mechanisms, Materials, and Devices.” S. S. Sun, N. S. Sariciftci (Editors), Taylor \& Francis Group LLC, Florida (2005).

4. C. Waldauf, P. Schilinsky, J. Hauch and C. J. Brabec, Thin Solid Films, 451-452, 503 (2004).

5. C. Li, Y. Chen, Y. Wang, Z. Iqbal, M. Chhowalla, and S. Mitra, J. Mater. Chem. 17, 2406 (2007).

6. A. F. Hepp, S. G. Bailey, and R. P. Raffaelle in Organic Photovoltaics. Mechanisms, Materials, and Devices. S. S. Sun, N. S. Sariciftci (Editors), Taylor \& Francis Group LLC, Florida (2005).

7. N. Spallanzani, C. A. Rozzi, D. Varsano, T. Baruah, M. R. Pederson, F. Manghi, and A. Rubio, J. Phys. Chem. B 113, 5345 (2009).

8. M. C. Scharber, D. Muhlbacher, M. Koppe, P. Denk, C. Waldauf, A. J. Heeger, and C. J. Brabec, Adv. Mater. 18, 789 (2006).

9. J. Brédas, J. E. Norton, J. Cornil, and V. Coropceanu, Acc. Chem. Res. 42, 1691 (2009).

10. L. J. K. Koster, V. D. Mihailetchi, and P. W. M. Blom, App. Phys. Lett. 88, 093511 (2006).

11. D. L. Cheung, and A. Troisi, J. Phys. Chem. C 114, 20479 (2010).

12. Y. Kanai and J. C. Grossman, Nano Lett. 7, 1967 (2007).

13. C. F. N. Marchiori and M. Koehler, Syn. Met. 160, 643 (2010).

14. J. J. M. Halls, J. Cornil, D. A. dos Santos, R. Silbey, D. H. Hwang, A. B. Holmes, J. L. 
Brédas, and R. H. Friend, Phys. Rev. B 60, 5721 (1999).

15. J. Piris, T. E. Dykstra, A. A. Bakulin, P. van Loosdrecgt, W. Knulst, M. T. Trinh, J. M. Schins, and L. D.A. Siebbeles, J. Phys. Chem. 113, 14500 (2009).

16. S. Cook, R. Katoh, and A. Furube, J. Phys. Chem. C 113, 2547 (2009).

17. M. Hallerman, S. Haneder, and E, Da Como, App. Phys. Lett 93, 053307 (2008).

18. T. Drori, C.-X. Sheng, A. Ndobe, S. Singh, J. Holt, and Z. V. Vardeny, Phys. Rev. Lett. 101, 037401 (2008).

19. E. Lioudakis, I. Alexandrou, and A. Othonos, Nanoscale Res. Lett. 4, 1475 (2009).

20. G. Grancini, D. Polli, D. Fazzi, J. Cabanillas-Gonzalez, G. Cerullo, and G. Lanzani, J. Phys. Chem. Lett. 2, 1099 (2011).

21. P. Vanlaeke. A. Swinnen, I. Haeldermans, G. Vanhoyland, T. Aernouts, D. Cheyns, C. Deibel, J. D’Haen, P. Heremans, J. Poortmans, and J. V. Manca, Sol. Energy Mater. Sol. Cells 90, 2150 (2006).

22. Y. Kim, S. Cook, S. M. Tuladhar, S. A. Choulis, J. Nelson, J. R. Durrant, D. C. Bradley, M. Giles, I. McCulloch, C. Ha, and M. Ree, Nature 5, 197 (2006).

23. B. Peng, Xia Guo, Y. C. Pan, and Y. Li, J. Phys. D: Appl. Phys. 44, 365101 (2011).

24. E. D. Gomez, K. P. Barteu, H. Wang, M. F. Toney, and Y. Loo, Chem. Commun. 47, 436 (2011).

25. E. Bundgaard and F. C. Krebs, Sol. Energy Mat. Sol. Cells 91, 954 (2007).

26. J. Zhao, A. Swinnen, G. Van Assche, J. Manca, D. Vanderzande, and B. Van Mele, J. Phys. Chem. B 113, 1587 (2009).

27. K. Inoue, R. Ulbricht, P. C. Madakasira, W. M. Sampson, S. Lee, Jose Gutierrez, J. Ferraris, and A. Zakhidov, Syn. Met. 154, 41 (2005).

28. G. Janssen, A. Aguirre, E. Goovaerts, P. Vanlaeke, J. Poortmans, and J. Manca, Eur. Phys. J. Appl. Phys. 37, 287 (2007).

29. K. Vakhshouri, S. VajjalaKesava, D. R.Kozub, E. D.Gomez, Mater. Lett. 90, 97 (2013). 
30. W. J. D. Beenken, F. Herrmann, M. Presselt, H. Hoppe, S. Shokhovets, G. Gobsch and E. Runge , Phys. Chem. Chem. Phys. (2014) DOI: 10.1039/c3cp42236d

31. T. Liu and A. Troisi, J. Phys. Chem. C 115, 2406 (2011).

32. S. Grimme, J. Anthony, T. Schwabe, and K. Muck-Lichtenfeld, Org. Biomol. Chem. 5, 741 (2007).

33. S. Grimme, J. Anthony, S. Ehrlich, and H. Krieg, J. Chem. Phys. 132, 154104 (2010).

34. J. P. Perdew, K. Burke, and M. Ernzerhof, Phys. Rev. Lett. 77, 3865 (1996).

35. ADF 2013. E. J. Baerends, J. Autschbach, J. A. Berger, A. Berces, F. M. Bickelhaupt,C. Bo, P. L. de Boeij, P. M. Boerrigter, L. Cavallo, D. P. Chong, L.Deng, R. M. Dickson, D. E. Ellis, M. van Faassen, L. Fan, T. H. Fischer, C.Fonseca Guerra, S. J. A. van Gisbergen, A. W. Götz, J. A. Groeneveld, O. V.Gritsenko, M. Gru “ ning, F. E. Harris, P. van den Hoek, C. R. Jacob, H. Jacobsen,L. Jensen, E. S. Kadantsev, G. van Kessel, R. Klooster, F. Kootstra, M.V. Krykunov, E. van Lenthe, J. N. Louwen, D. A. McCormack, A. Michalak,J. Neugebauer, V. P. Nicu, V. P. Osinga, S. Patchkovskii, P. H. T. Philipsen, D. Post, C. C. Pye, W. Ravenek, J. I. Rodríguez, P. Romaniello, P. Ros, P. R. T. Schipper, G. Schreckenbach, J. G. Snijders, M. Sola, M. Swart, D. Swerhone, G. te Velde, P. Vernooijs, L. Versluis, L. Visscher, O. Visser, F. Wang, T. A. Wesolowski, E. M. van Wezenbeek, G. Wiesenekker, S. K. Wolff, T. K. Woo, A. L. Yakovlev, and T. Ziegler. ADF 2013. Theoretical Chemistry, Vrije Universiteit: Amsterdam, The Netherlands. Available at: http://www.scm.com. Acessed on April 7, 2014.

36. C. F. Guerra, J. G. Snijders, G. te Velde, and E. J. Baerends, Theor. Chem. Acc. 99, 391 (1998).

37. G. te Velde, F. M. Bickelhaupt, S. J. A. van Gisbergen, C. Fonseca Guerra, E. J. Baerends, J. G. Snijders, and T. Ziegler, J. Comput. Chem. 22, 931 (2001).

38. B. C. Thompson and J. M. J. Fréchet, Angew. Chem. Int. Ed. 47, 58 (2008).

39. P. Mori-Sanchez, A.J. Cohen, W.T. Yang, Phys. Rev. Lett. 100, 146401 (2008).

40. X. Zheng, A.J. Cohen, P. Mori-Sanchez, X.Q. Hu, W.T. Yang, Phys. Rev. Lett. 107, 026403 (2011). 
41. M.J.G. Peach, P. Benfield, T. Helgaker, D.J. Tozer, J. Chem. Phys., 128, 044118 (2008).

42. M.J.G. Peach, C.R. Le Sueur, K. Ruud, M. Guillaume, D.J. Tozer, Phys. Chem. Chem. Phys. 11 , 4465-4470 (2009).

43. S. Cook, H. Ohkita, Y. Kim, J. J. Benson-Smith, D. D.C. Bradley, J. R. Durrant, Chem. Phy. Lett. 445, 276-280 (2007).

44. G. Li, V. Shrotriya, Y. Yao, J. Huang, and Y. Yang, J. Mater. Chem. 17, 3126 (2007).

45. V. Shrotriya, J. Ouyang, R. J. Tseng, G. Li, Y. Yang, Chem. Phys. Lett. 411, 138 (2005).

46. Bin Yin, Liying Yang, Yongsheng Liu, Yongsheng Chen, Qingjin Qi, Fengling Zhang, and Shougen Yin, App. Phys. Lett. 97, 023303 (2010).

47. J. Guay, P. Kasai, A. Dim, Ruilian Wu, James M. Tour and Le H. Dao, Chem. Mater. 4, 1097-1105, (1992).

48. D. Chirvase, J. Parisi, J.C. Hummelen, V. Dyakonov, Nanotechnology 15, 1317 (2004).

49. J. I. Rodríguez, R. F. W. Bader, P. W. Ayers, C. Michel, A. Götz, C. Bo, Chem. Phys. Lett., 472, 149 (2009).

50. E. Sanville, S.D. Kenny, R. Smith, G. Henkelman, J. Comput. Chem. 28, 899 (2007). 


\section{Figure Captions}

Figure 1. The structure of the P3HT-PCBM dimer for isomer 1 (upper row) and isomer 2 (lower row). (a) initial (or guess) geometries used in the geometry optimization procedure; (b) optimized geometries; (c) the same optimized as in (b) but now hiding the alkane side chains in P3HT and hydrogen atoms. The energy difference between the two isomer is equal to $3.126 \mathrm{eV}$.

Figure 2. Interatomic distances histogram between P3HT and PCBM atoms in-the-dimer are shown for (a) Isomer 1 and (b) Isomer 2. The corresponding dimer isomer geometries as long as the minimum $\left(\mathrm{h}_{\min }\right)$, maximum $\left(\mathrm{h}_{\max }\right)$ and average $\left(\mathrm{h}_{\min }\right)$ distances are shown inset.

Figure 3. Diagrams with the dihedral angle values (in degrees) between adjacent thiophene rigns of P3HT in-the-dimer for (a) Isomer 1, and (b) Isomer 2.

Figure 4. Main electronic transitions in (a) dimer Isomer 1 and (b) dimer Isomer 2.

Figure 5. Absorption spectra for the isolated P3HT (red line), isolated PCBM (black line), P3HTPCBM dimer Isomer 1 (green line) and Isomer 2 (blue line) obtained via TDDFT calculations at the DFT optimized geometries. The absorption spectrum of isolated PCBM can be seen in more detail in the inset. 
a)
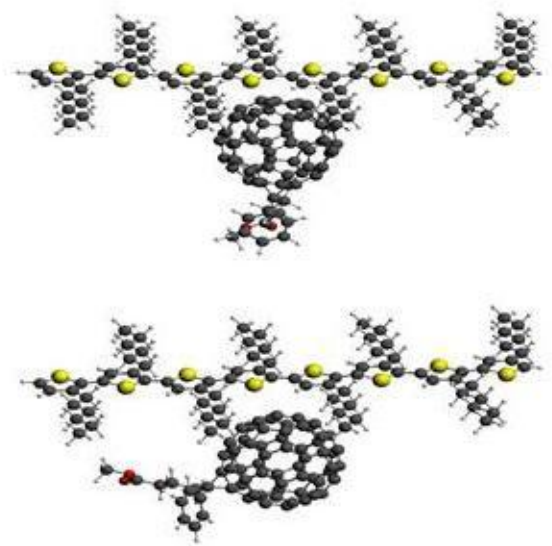

) b)
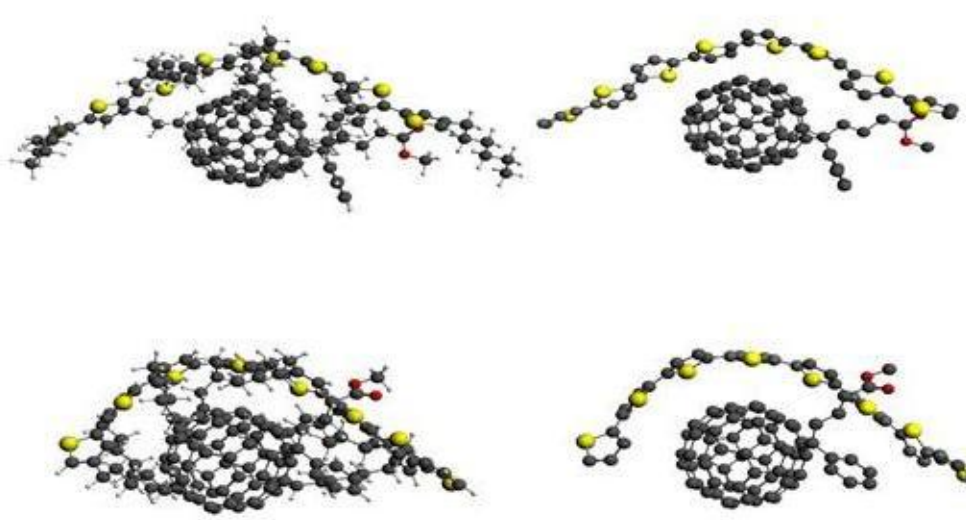

) c)

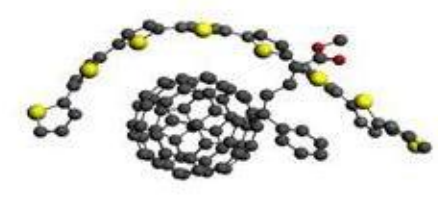

)

$$
\text { . }
$$

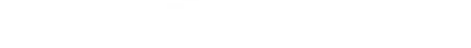

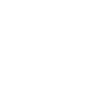


a)

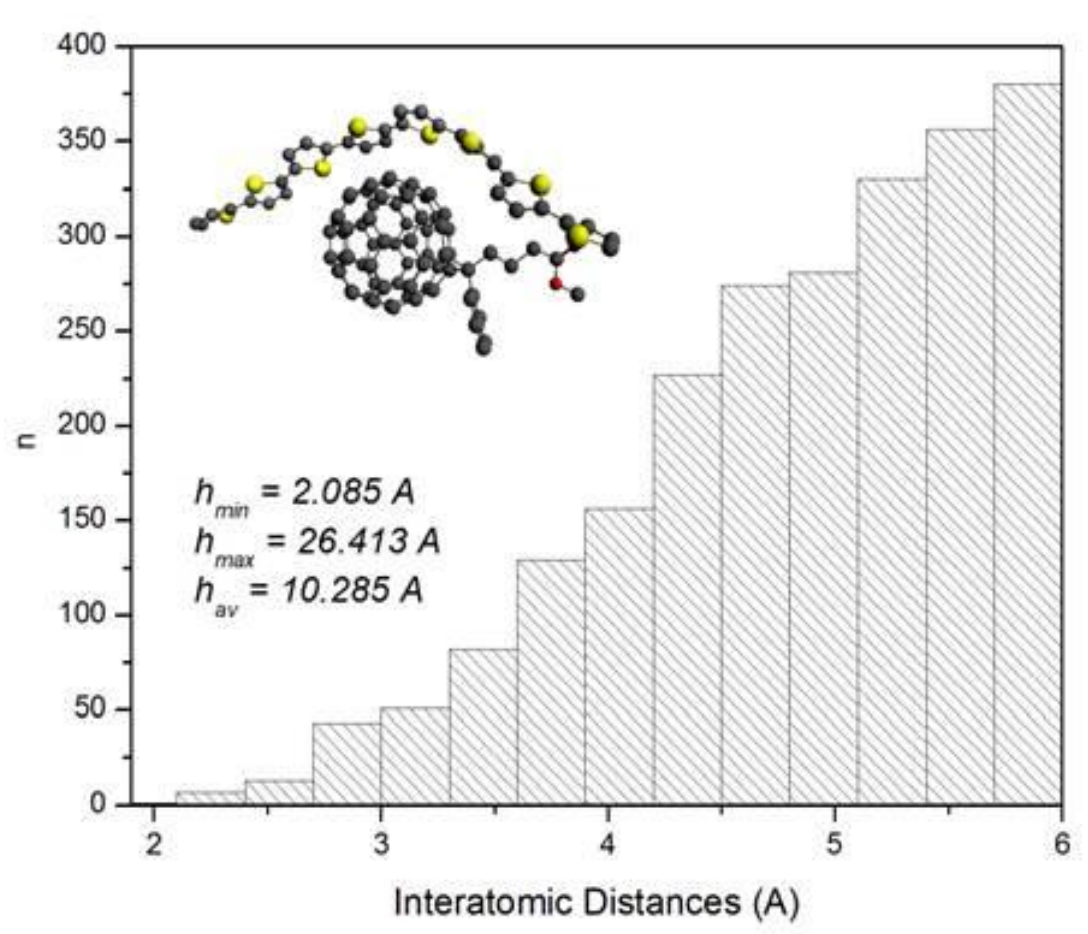

b)

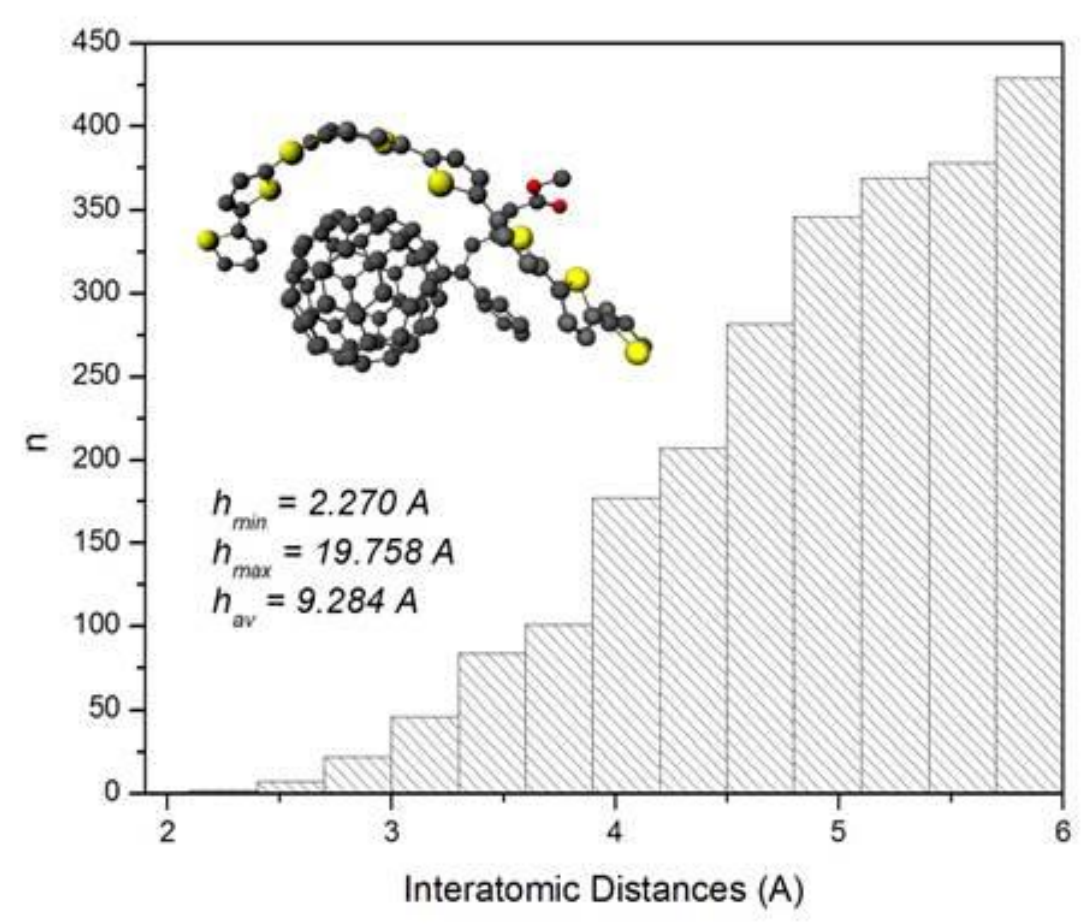

Figure 2 

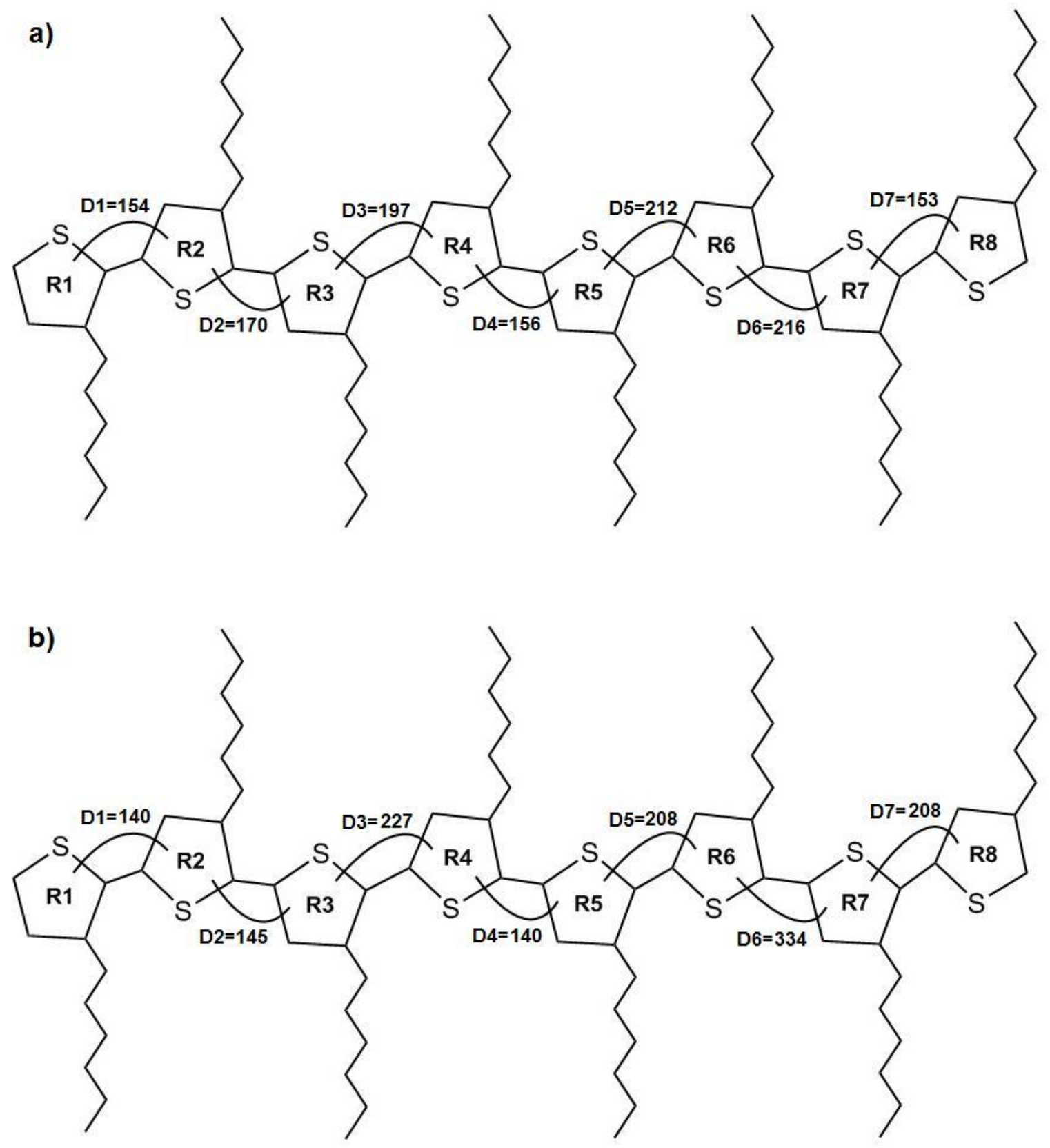
a)

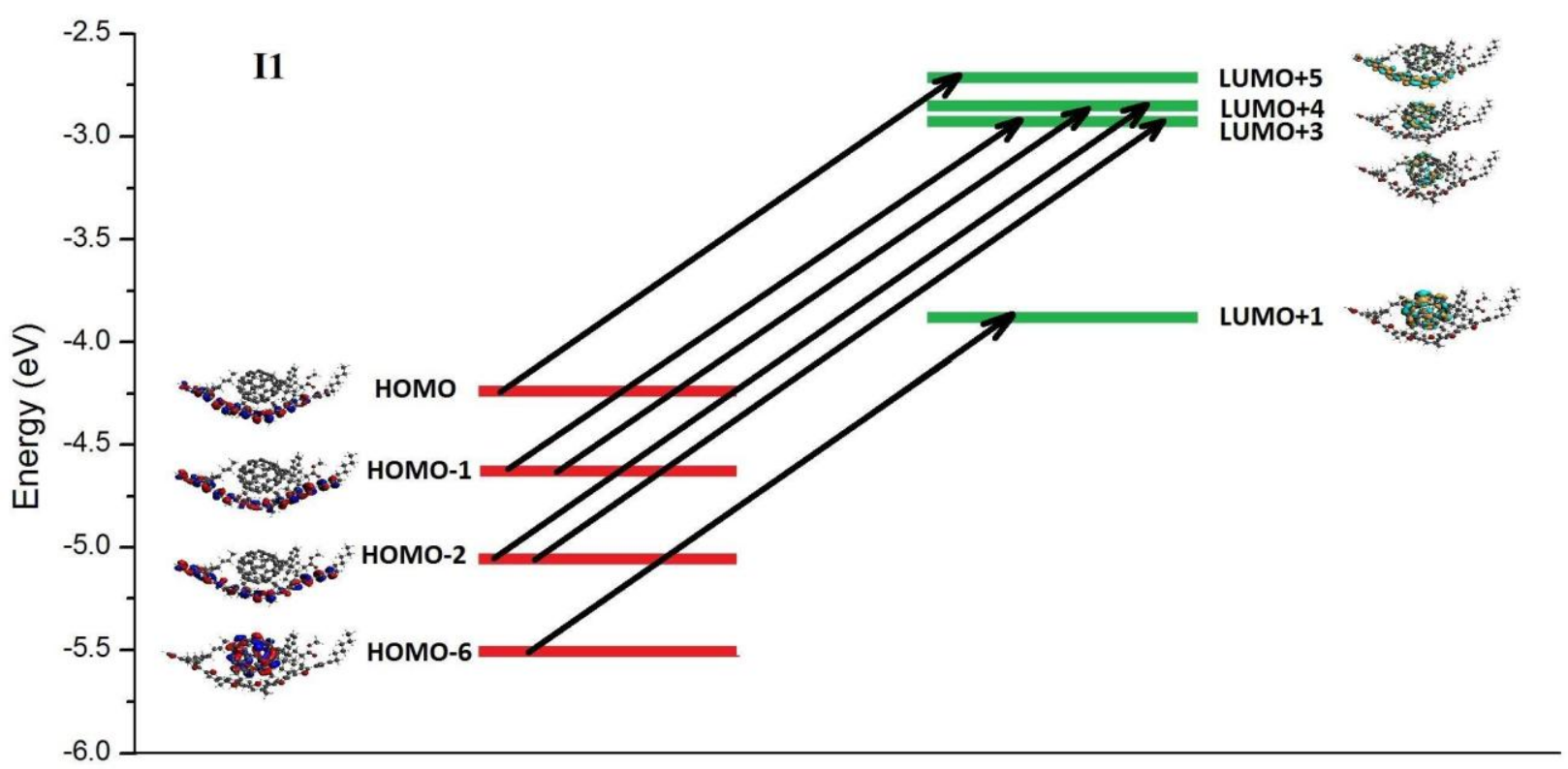

b)

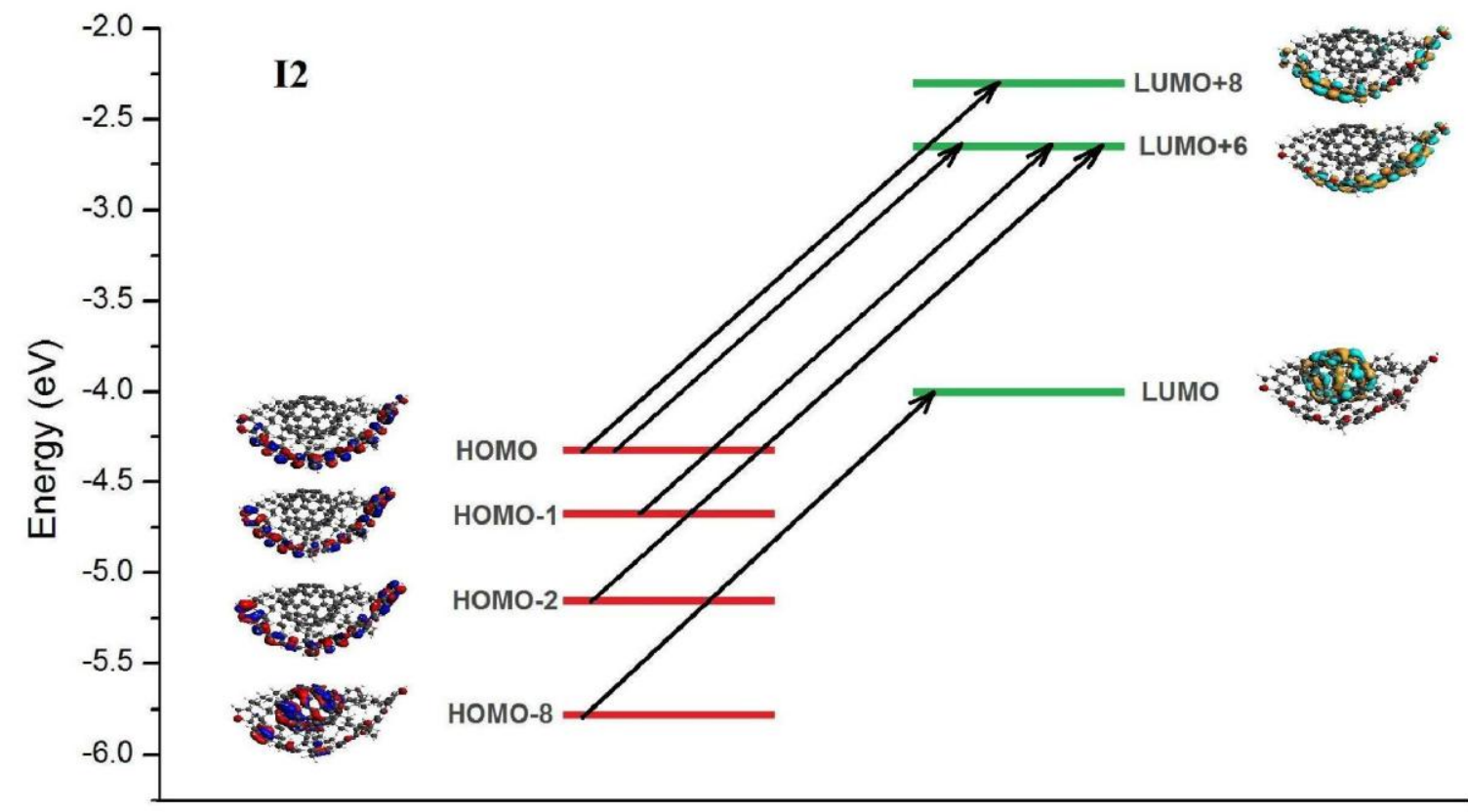




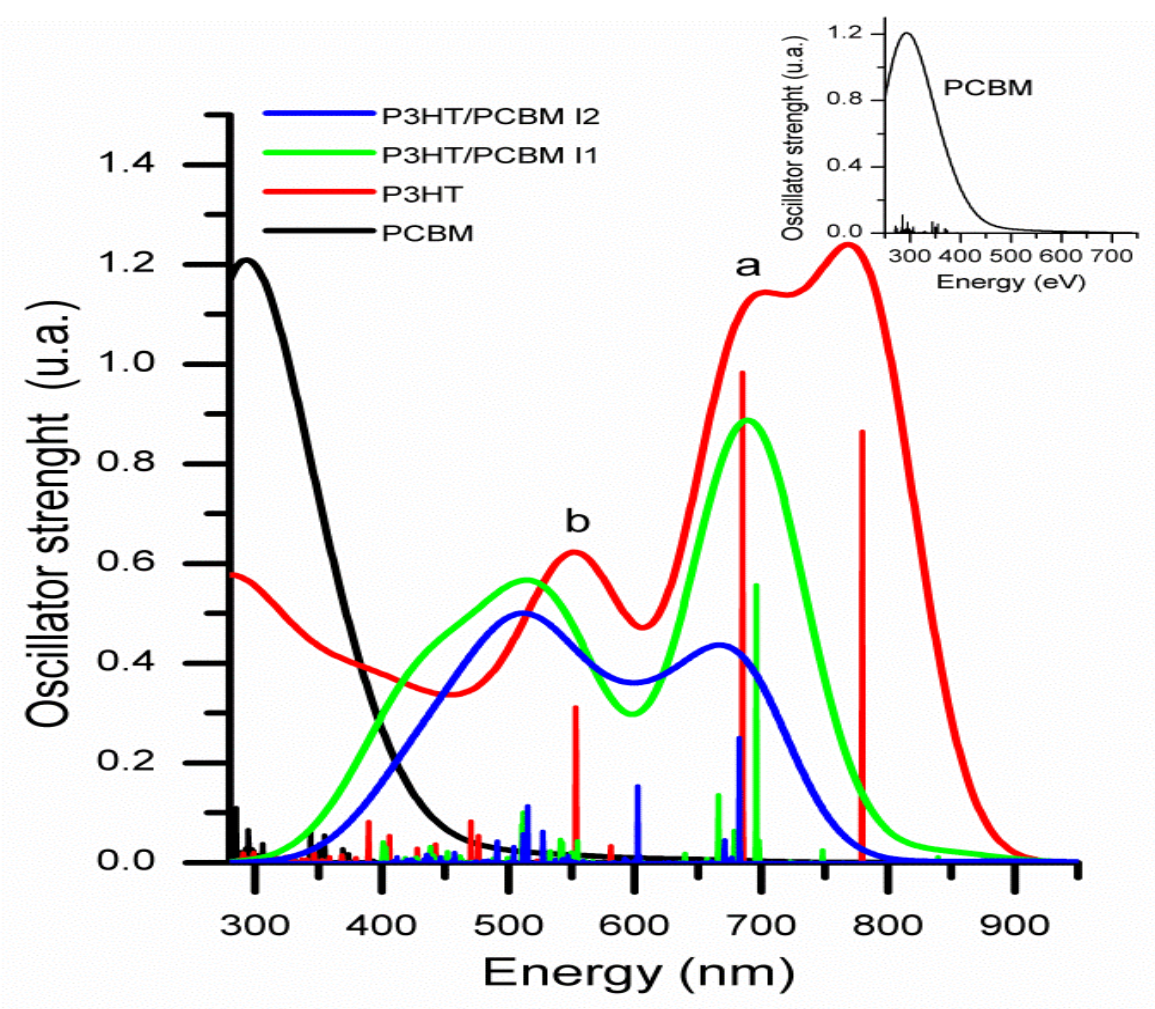

Figure 5. 
a)

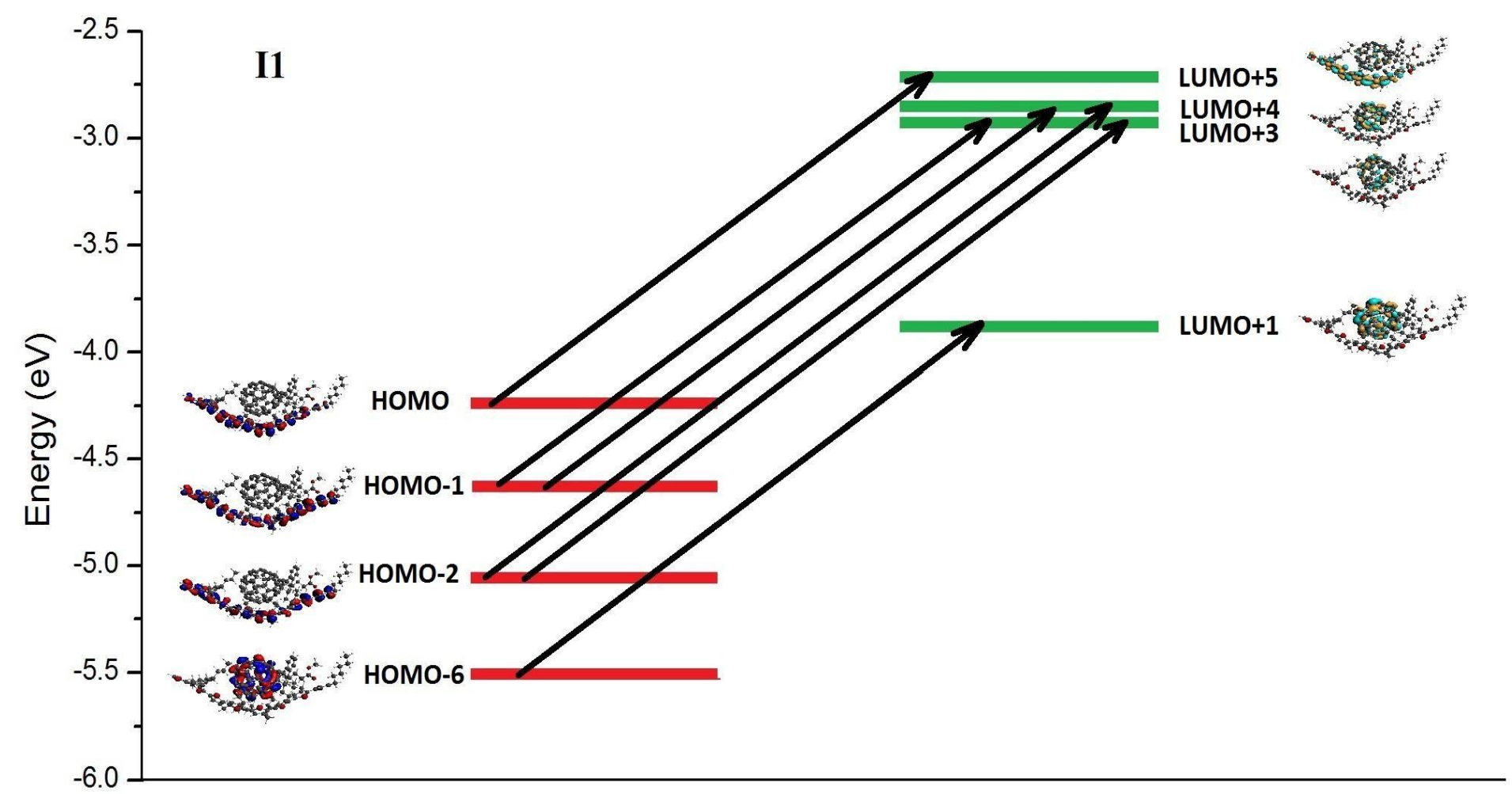

b)

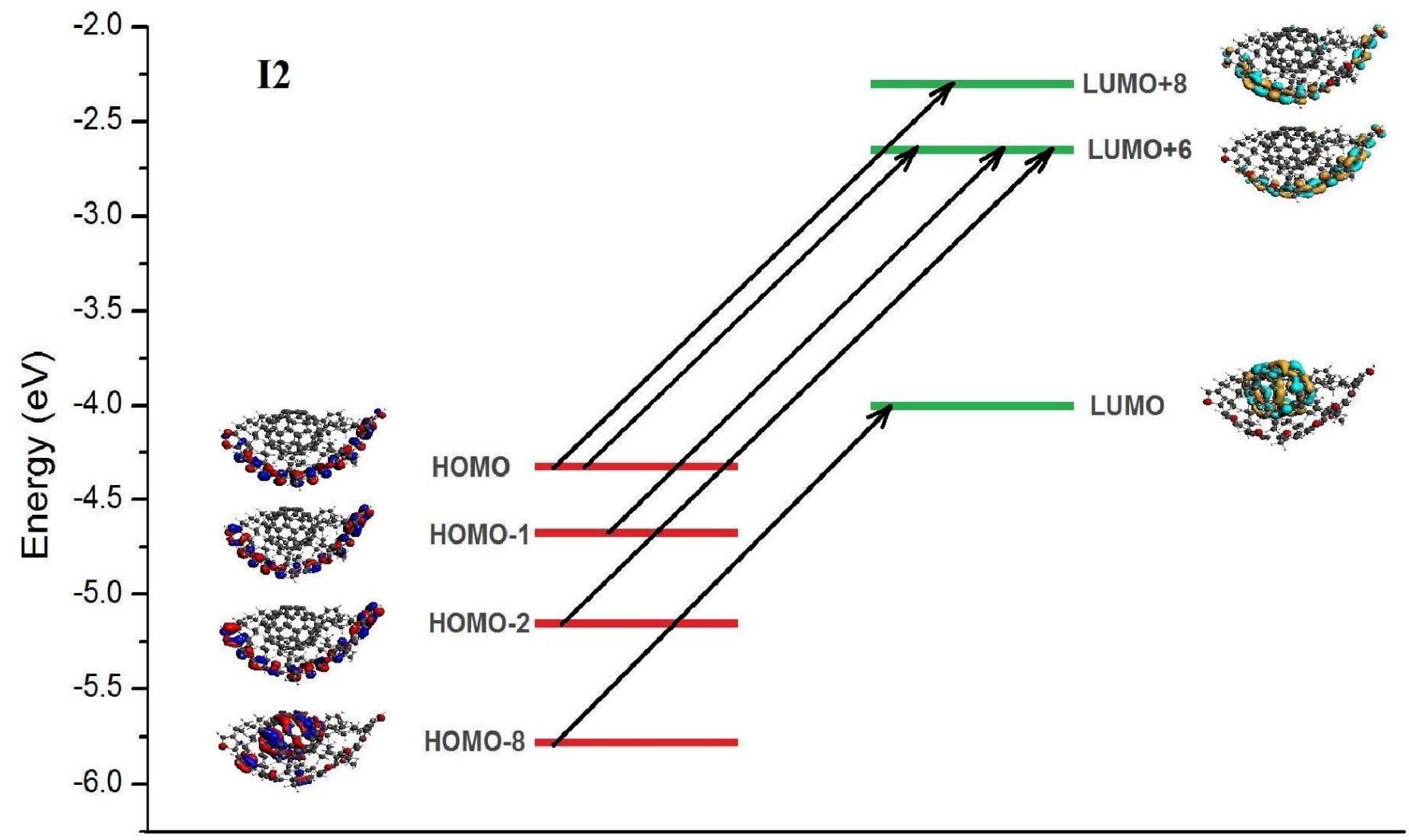

